Article

\title{
Color-Tunable Organic Light Emitting Diodes for Deep Blue Emission by Regulating the Optical Micro-Cavity
}

\author{
Jixin Jiang ${ }^{1,2}$, Weiye Zheng ${ }^{1,2}$, Junfei Chen ${ }^{1,2}$, Zheng $\mathrm{Xu}^{1,2}$, Dandan Song ${ }^{1,2}$, Bo Qiao ${ }^{1,2}$ \\ and Suling Zhao ${ }^{1,2, * \mathbb{D}}$ \\ 1 Key Laboratory of Luminescence and Optical Information, Ministry of Education, Beijing Jiaotong University, \\ Beijing 100044, China; 19118027@bjtu.edu.cn (J.J.); 16118445@bjtu.edu.cn (W.Z.); 17118458@bjtu.edu.cn (J.C.); \\ zhengxu@bjtu.edu.cn (Z.X.); ddsong@bjtu.edu.cn (D.S.); bqiao@bjtu.edu.cn (B.Q.) \\ 2 Institute of Optoelectronics Technology, Beijing Jiaotong University, Beijing 100044, China \\ * Correspondence: slzhao@bjtu.edu.cn; Tel./Fax: +86-10-51684858
}

Academic Editor: Jwo-Huei Jou

Received: 26 May 2020; Accepted: 20 June 2020; Published: 22 June 2020

\begin{abstract}
Nowadays, most blue organic light emitting diodes (OLEDs) are fabricated by using sky-blue emitters which are more easily synthesized when compared with other deep blue emitters. Herein, we put forward a new idea of using an optical micro-cavity based on metal electrodes to regulate electroluminance (EL) spectra of sky-blue organic light emitting diodes to obtain a saturated deep blue emission with a narrowed full-width at half-maximum (FWHM). First, we simulate micro-cavity OLEDs and find that the transmission of the anode plays an important role in the forward emission. Meanwhile, the optical path of micro-cavity OLEDs as well as the phase shifting from electrodes influence the EL spectra and induce the extra intensity enhancement. The results show that when the resonant cavity optical path is regulated by changing the thickness of emitting layer (EML) from $25 \mathrm{~nm}$ to $75 \mathrm{~nm}$ in the micro-cavity, the EL peak of blue OLEDs has a redshift from $479 \mathrm{~nm}$ to $493 \mathrm{~nm}$ with FWHM shifting from $69.8 \mathrm{~nm}$ to $83.2 \mathrm{~nm}$, when compared to the device without the micro-cavity, whose approximate EL peak and FWHM are $487 \mathrm{~nm}$ and $87 \mathrm{~nm}$, respectively. However, the efficiency of electroluminescence decreases in micro-cavity OLEDs. We speculate that this is on account of the ohmic contact between ITO and Ag, the surface plasma effect and the rough morphology induced by Ag electrodes.
\end{abstract}

Keywords: micro-cavity; organic light emitting diodes; blue emission; spectral shift

\section{Introduction}

As we all know, organic light-emitting diodes (OLEDs) have become the new generation of flat-panel display and solid-state light source for their excellent properties, which include solid-state active emitting-light and thinness, and they attract people's attention for the rapid advancement and significant progress of OLEDs [1-4]. In particular, the property improvement of blue OLEDs is the key technique for achieving a better performance of OLEDs for their applications. Deep blue OLEDs are essential to flat-panel displays, mainly for reducing power consumption and saturated color performance. According to the National Television System Committee or the European Broadcasting Union (EBU) standard, deep blue color is usually defined as the blue electroluminescence (EL) emission with a Commission International de L'Eclairage (CIE) coordinate of $\mathrm{y}<0.08$ or $\mathrm{y}<0.06$ respectively $[5,6]$. To obtain saturated deep blue organic light emitting diodes, many studies are conducted in molecule design [7-10]. More recently, Li et al. designed a novel bipolar carbazole/phenanthroimidazole derivative named CzB-MOPPI, whose non-doped devices show the emission at $435 \mathrm{~nm}$ with a CIE 
of $(0.16,0.08)$, a maximum luminance of $6450 \mathrm{~cd} / \mathrm{m}^{2}$, a maximum current efficiency (CE) of $3.34 \mathrm{~cd} / \mathrm{A}$ and an external quantum efficiency (EQE) as high as 5.97\% [11]. Kondo et al. reported a thermally activated delayed-fluorescence material that exhibits ultrapure blue emission, whose devices emit light at $469 \mathrm{~nm}$ with a full-width at half-maximum of $18 \mathrm{~nm}$ with an external quantum efficiency of $34.4 \%$ at the maximum and $26.0 \%$ at $1000 \mathrm{~cd} \mathrm{~m}^{-2}$ [12]. However, all these saturated deep-blue organic materials for full-color flat-panel displays have a deep energy level (HOMO) and a very wide band-gap $[13,14]$. It is quite difficult to design their molecular structure with a good carrier mobility and environment stability [15]. Among the various known blue organic materials, sky-blue emitters are considered to be the main molecule design [16-18]. Thus, we put forward a new idea to investigate a device structure to obtain the saturated deep blue emission based on sky-blue organic light-emitting diodes by introducing an optical cavity in devices.

As is known, an optical cavity can be formed in OLEDs by using high refractive index metallic electrodes [19-23] or DBR [24-26] composed of semi-conductors with a low and high ordered refractive index arrangement. Generally, metal electrodes are the preferred choices for their easy fabrication. Optical waves radiated from dipoles in the emitting layer (EML) reflect when they get to electrodes, which are regarded as parallel mirrors, and then following reflected light beams are subjected to the Fabry-Pérot interference. In other words, using electrodes can introduce the electromagnetic boundary conditions, and the photon density states can be altered strongly, with the states near the cavity resonance mode being enhanced while the others are weakened. This shows that the optical cavity in OLEDs enhances the emission solely at a particular color and suppresses others in the meantime, which is called micro-cavity. Li et al. reported that the micro-cavity structure markedly improves the efficiencies of green organic light-emitting diodes with a spectral peak intensity 4.3 times higher than that in a conventional device and a two-fold enhancement of the current efficiency [21], while the luminance enhancements of blue and red micro-cavity devices are small.

Here, we analyzed an optical resonant cavity, that is a micro-cavity in sky-blue OLEDs, on a theoretical basis to provide a convenient solution to saturated deep blue OLEDs. The results show that the emission of sky-blue OLEDs could be redistributed and that the spectra could be blue-shifted to the desired emission due to the multiple-beams interference and wide-angle interference by optimizing the device parameters. By introducing the micro-cavity effect into shy-blue OLEDs experimentally, we demonstrated that micro-cavity OLEDs possess a blue shift of the EL peak from $493 \mathrm{~nm}$ to $479 \mathrm{~nm}$ with a narrowing of the FWHM to $69.8 \mathrm{~nm}$ when compared to non-cavity devices with an approximate EL peak and FWHM of $487 \mathrm{~nm}$ and $87 \mathrm{~nm}$, respectively, at the detriment of the luminance and current efficiency (CE).

\section{Results and Discussion}

\subsection{Optical Properties of the Micro-Cavity in OLEDs}

It is reported that the micro-cavity is usually used in green OLEDs to enhance their intensity and efficiency by keeping the electroluminescence peak of emitters and resonance wavelength unchanged at the same time. Here, we use the micro-cavity to regulate the spectra of blue devices by changing the mode indexes. A multi-layer structure exists in bottom-emitting OLEDs (BOLEDs). Total reflection occurs on the internal surface of the cathode for its high reflectivity and thickness; as for the anode, semi-reflection occurs on it. This means that the emission of radiative dipoles reaches metal electrodes and produces a multi-beam interference with a wide-angle. The micro-cavity introduces the wide-angle interference and multi-beam interference into BOLED at the same time, as shown in Figure 1. Figure 1A shows wide-angle interference in micro-cavity bottom-emitting OLEDs and multi-beam interference is shown in Figure 1B. As shown in Figure $1, E_{1}$ represents the emission in the EML; $\theta_{0}$ and $\theta_{1}$ are incident angle and refraction angle on electrodes, respectively; $\mathrm{L}_{1}, \mathrm{~L}_{2}$, $\mathrm{L}$ are the optical path from EML to two electrodes and between two electrodes, respectively. 
A)

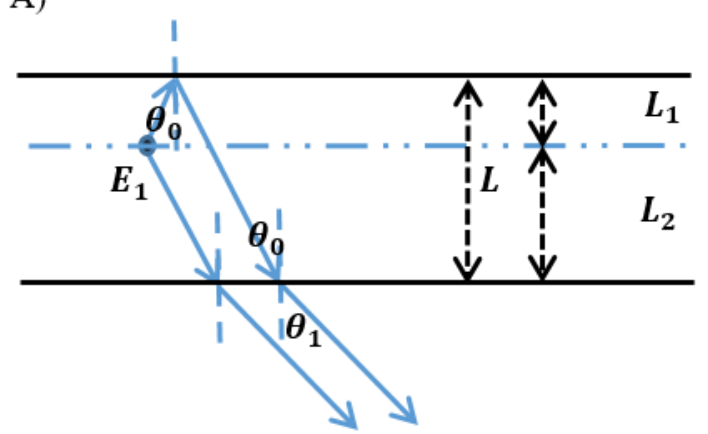

B)



Figure 1. (A) Wide-angle interference and (B) multi-beam interference in micro-cavity bottom-emitting OLEDs. $E_{1}$ represents the emission in the EML; $\theta_{0}$ and $\theta_{1}$ are incident angle and refraction angle on electrodes, respectively; $\mathrm{L}_{1}, \mathrm{~L}_{2}, \mathrm{~L}$ are the optical path from EML to two electrodes and between two electrodes, respectively.

In order to analyze the effect of this micro-cavity in BOLEDs, we implemented a theoretical simulation of its mechanism and found that the transmission of the anode plays an important role in the forward emission. Here, micro-cavity OLEDs can be simplified as a Fabry-Pérot resonator. In the resonator, a light source exists in the emitting layer between the parallel electrodes, as shown in Figure 2.

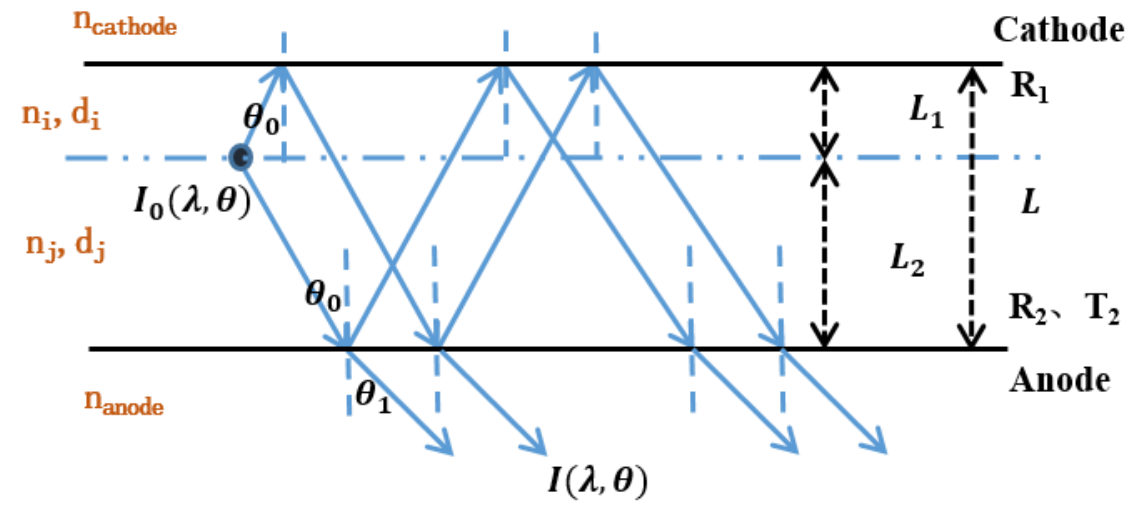

Figure 2. Micro-cavity OLEDs schematic.

Assuming $I_{0}(\lambda, \theta)$ as the emission of radiating dipoles in the emitting layer, after the wide-angle and multi-beam interferences inside the cavity, the light finally emits through the anode, which is semi-transparent. Then, the dividing amplitude is used to calculate the forward emission. The intensity of the forward emission $I(\lambda, \theta)$ from the micro-cavity has been calculated via Equations (1)-(4) [27-30]:

$$
\begin{gathered}
I(\lambda, \theta)=\frac{T_{2}\left(1+R_{1}+2 \sqrt{R_{1}} \cos \left(\frac{4 \pi L_{1}}{\lambda}-\varphi_{1}\right)\right)}{1+R_{1} R_{2}-2 \sqrt{R_{2} R_{1}} \cos \left(\frac{4 \pi L}{\lambda}-\varphi_{1}-\varphi_{2}\right)} I_{0}(\lambda, \theta) \\
L=L_{1}+L_{2}=\sum n_{i} d_{i}+\sum n_{j} d_{j} \\
L_{1}=\sum n_{i} d_{i} \\
L_{2}=\sum n_{j} d_{j} \\
\varphi_{1}, \varphi_{2}, T_{2}, R_{1}, R_{2}=f(\lambda, \theta)
\end{gathered}
$$


Here, $R_{1}$ and $R_{2}$ are the reflective index of the cathode and anode, respectively, and $T_{2}$ is the transmittance of the anode. $\varphi_{1}$ and $\varphi_{2}$ are the phase-shift angle from the cathode and anode, respectively, $n_{j}$ and $n_{i}$, are the refractive index of each organic layer between the EML and anode or cathode, respectively, and, similarly, $d_{j}$ and $d_{i}$ are their thicknesses; $\mathrm{n}_{\text {anode }}$ and $\mathrm{n}_{\text {cathode }}$ are the refractive index of the anode and cathode, respectively. $L_{1}, L_{2}$ and $L$ are the optical path from the radiative dipoles to the cathode, to the anode and between two metal electrodes, respectively.

In BOLEDs, the plane light is emitted from the emitting layer and incidents normal to electrodes. The reflectivity and transmissivity of metal electrodes can be calculated by the single-layer transfer matrix, which is obtained by Maxwell's equations. Maxwell's equations use four field quantities D, E, $\mathrm{B}, \mathrm{H}$ and two sources $\mathrm{J}, \rho$ to demonstrate the variation of the electromagnetic field with the transfer matrix and thus to obtain a characteristic equation of the medium, which we can use for its optical constants, as in the following Equations (6)-(14):

$$
\begin{gathered}
M_{m}=\left[\begin{array}{cc}
A & B \\
C & D
\end{array}\right]=\left[\begin{array}{cc}
\cos \delta_{m} & \frac{i}{\eta_{m}} \sin \delta_{m} \\
\mathrm{i} \eta_{m} \sin \delta_{m} & \cos \delta_{m}
\end{array}\right] \\
\eta_{m}=N_{m} y_{0} \\
y_{0}=\sqrt{\frac{\varepsilon_{0}}{\mu_{0}}} \\
\delta_{m}=\frac{2 \pi}{\lambda} N_{m} d_{m} \cos \theta_{m} \\
\mathrm{r}=\frac{A \eta_{m}+B \eta_{m} \eta_{\text {air }}-C-D \eta_{\text {air }}}{A \eta_{m}+B \eta_{m} \eta_{\text {air }}+C+D \eta_{\text {air }}}=x+i y \\
\mathrm{t}=\frac{2 \eta_{m}}{A \eta_{m}+B \eta_{m} \eta_{\text {air }}+C+D \eta_{\text {air }}} \\
R_{m}=r \cdot r^{*} \\
T_{m}=\frac{\eta_{\text {air }}}{\eta_{m}} t \cdot t^{*} \\
\varphi_{r m}=\tan ^{-1}\left[\frac{y}{x}\right]
\end{gathered}
$$

By Equations (11)-(13), we can obtain the reflectivity $R_{m}$, transmissivity $T_{m}$ of the metallic electrode and its phase-shift $\varphi_{r m}$. For the metallic electrode, $\mathrm{M}_{\mathrm{m}}$ is the transfer matrix, and $\left[\begin{array}{l}B \\ C\end{array}\right]$ is its characteristic equation. $\mathrm{r}$ and $\mathrm{t}$ are the reflection coefficient and the transmission coefficient, respectively. $\delta_{m}$ is the optical path of the electrode, and $\eta_{m}, \eta_{\text {air }}$ are the optical admittance of medium and air. $y_{0}$ is the optical admittance of free space, which can be considered as a unit. $\varepsilon_{0}, \mu_{0}$ is the permittivity and magnetic conductivity of vacuum. $d_{m}$ is the thickness of the electrode, and $N_{m}$ is the complex refractive index of metal including the refractive index $\mathrm{n}$ and extinction coefficient $\mathrm{k}$ and is calculated as in the following Equations (15) and (16):

$$
\begin{aligned}
& \varepsilon(\omega)=\varepsilon_{1}(\omega)+i \varepsilon_{2}(\omega) \\
& N_{m}=n+i \kappa=\sqrt{\varepsilon(\omega)}
\end{aligned}
$$

The complex dielectric function (14) can describe the optical properties of the medium, and $\varepsilon(\omega)$ is usually called the dielectric constant, where $(\omega)$ and $(\omega)$ are referred from The Handbook on Optical Constants of Metals to calculate the $\mathrm{N}_{\mathrm{m}}$ of the metal.

Figure 1 shows that the multi-beam interference and the wide-angle interference have a co-effect on the dipole emission. The enhancement generated at the same time from both two types of interference 
can be narrower and strengthen the forward emission. Equations (17) and (18) make clear the condition for the interference enhancement:

$$
\begin{gathered}
\frac{4 \pi L_{1}}{\lambda}-\varphi_{1}=2 k \pi \\
\frac{4 \pi L}{\lambda}-\varphi_{1}-\varphi_{2}=2 k^{\prime} \pi \\
k, k^{\prime}=0,1,2,3 \ldots
\end{gathered}
$$

Here, $k, k^{\prime}$ are the mode indexes corresponding to the resonance wavelengths. If the thickness of the organic layers is fixed as a constant, metallic electrodes play important parts in the micro-cavity effect for regulating the spectra of micro-cavity OLEDs precisely. Obviously, in a single resonant cavity whose cavity length is fixed, only one resonance wavelength can be emitted, and it is impossible to optimize the micro-cavity effects for different colors in a single resonant cavity.

In order to enhance the effect of Fabry-Pérot in a micro-cavity, a front semitransparent electrode with a high reflectivity and low absorption loss in the visible range is preferred, and Ag is mostly commonly used as the electrode to form a Fabry-Pérot cavity in OLEDs, due to its low extinction coefficient $(\mathrm{k})$ value and simple fabrication steps. In addition, it is not easily oxidized in the air, and its high work function matches better with the HOMO level of the hole injection layer than other metals. In our experiments, the cathode material is aluminum $(\mathrm{Al})$, and the optical simulation is carried out by using Matlab R2018a. According to Equations (6)-(14), we calculated the transfer matrixes of metallic electrodes and their phase shifts based on the Handbook of Optical Constants of Metals.

\subsection{Design of the Micro-Cavity in Blue OLEDs}

In order to obtain efficient blue OLEDs, 10-(4-(4,6-diphenyl-1,3,5-triazin-2-yl)phenyl)-10H -spiro [acridine-9,9'-fluorene] (SpiroAC-TRZ) has been chosen as the sky-blue emitter, 3,3-di(9H-carbazol-9yl)biphenyl (mCBP) as the host, dipyrazino[2,3-f:2',3'-h ]quinoxaline-2,3,6,7,10,11-hexacarbonitrile (HAT-CN) as the hole transporting layer (HTL), di-[4-(N,N-di-p-tolyl-amino)-phenyl]cyclohexane (TAPC) as the exciton blocking layer (EBL), 2,2' ,2' '(1,3,5-benzenetriyl)tris-(1-phenyl-1H-benzimidazole) (TPBi) as the electron transporting layer (ETL) and lithium fluoride (LiF) as the electron injection layer (EIL) to match the energy level of each layer. Figure 3A-E shows the molecule structures of HAT-CN, TAPC, mCBP, SpiroAC-TRZ and TPBi, respectively.

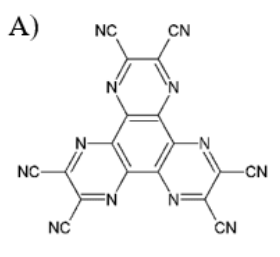

HAT-CN

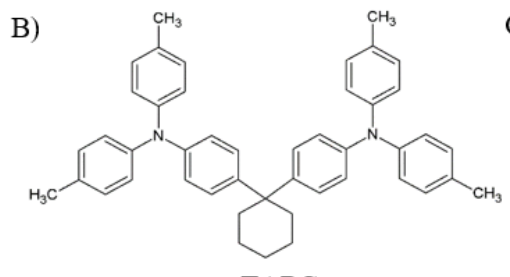

TAPC

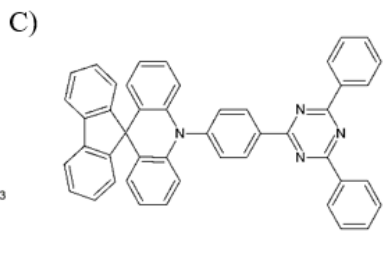

$\mathrm{mCBP}$
D)

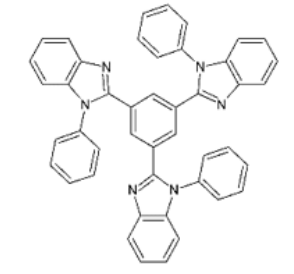

SpiroAC-TRZ
E)

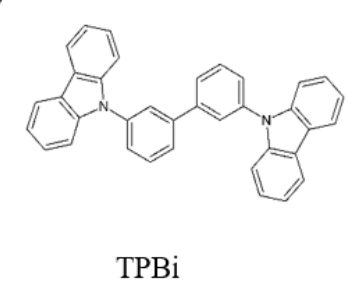

Figure 3. Molecule structure of organic materials: (A) HAT-CN, (B) TAPC, (C) mCBP, (D) SpiroAC-TRZ, (E) TPBi. 
Figure 4 shows the schematic diagram of the micro-cavity OLEDs: A) with metallic electrodes and the control devices B) without the semi-transparent anode, corresponding to the energy level of C) and D), respectively. The refractive indices of these organic semiconducting materials have similar values $\approx 1.7-1.9\left(\mathrm{n}_{\mathrm{org}} \approx 1.7 \sim 1.9\right)$ in the visual light spectral region. Two kinds of OLEDs were simulated via Equations (1)-(5), taking the transfer matrix into consideration.

A)



B)



C)

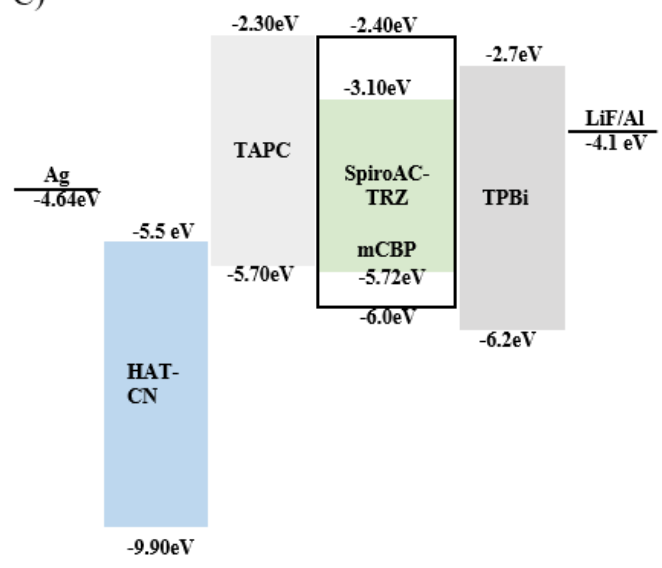

D)

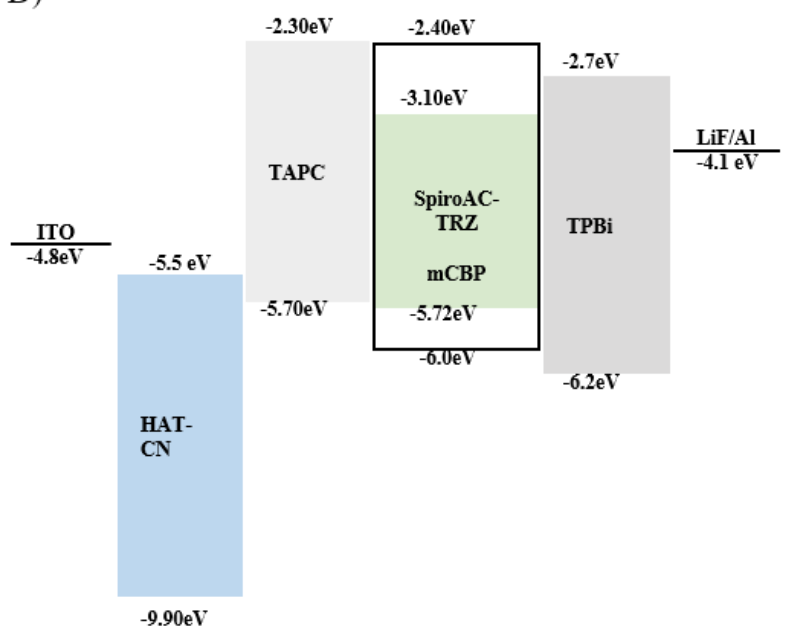

Figure 4. (A) Micro-cavity OLEDs device structure, (B) ITO-based OLEDs device structure, (C) Energy level of micro-cavity OLEDs, (D) Energy level of ITO-based OLEDs.

\subsection{Theoretical Simulation of the Micro-Cavity in Blue OLEDs}

According to the Handbook of Optical Constants of Metals by Sadao Adachi, we fit the curve of refractive indexes of $\mathrm{Ag}, \mathrm{Al}, \mathrm{Au}$ and $\mathrm{Mg}$ as shown in Figure $5 \mathrm{~A}, \mathrm{~B}$, with $\mathrm{n}$ and $\mathrm{k}$ as the ordinary refractive index and the extinction coefficient, respectively. The findings show that the absorption of $\mathrm{Au}$ is inferior to Ag, which means less optical loss. Thus, we simulate models of microcavities with $\mathrm{Au}$ and $\mathrm{Ag}$ layers with different thicknesses, respectively, where the optical length is set as 120 $\left(\mathrm{n}_{\text {org }} \approx 1.7 \sim 1.9\right.$ ), as shown in Figure 5 C,D. $I_{0}$ is the emission of radiating dipoles in the emitting layer. It is apparent that Au-based electrodes can enhance the luminance intensity. However, as shown in Figure 5E,F, the Au layer is not suitable for the electrode because we need an ultrathin OLED for which the optical path between two electrodes is just 40 to make the peak wavelength be blue shifting; otherwise, in a conventional OLED where the optical path is 240 , we will obtain the second peak, meaning approximately $170 \mathrm{~nm}$-thick organic layers between electrodes. As a result, Ag is chosen as the electrode for the micro-cavity OLED. 
A)
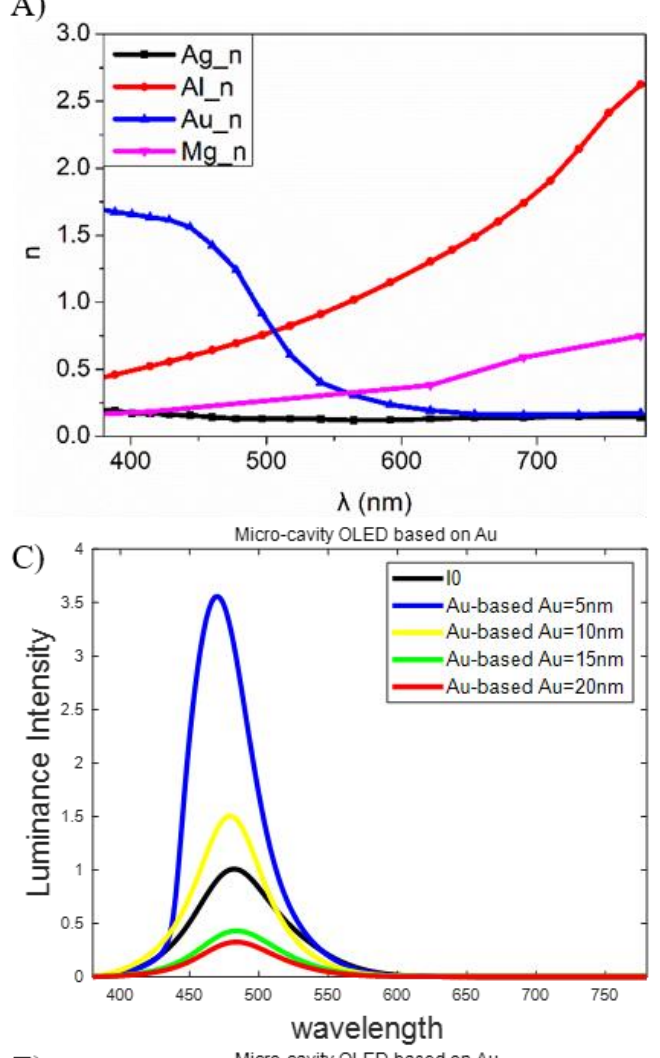

E)

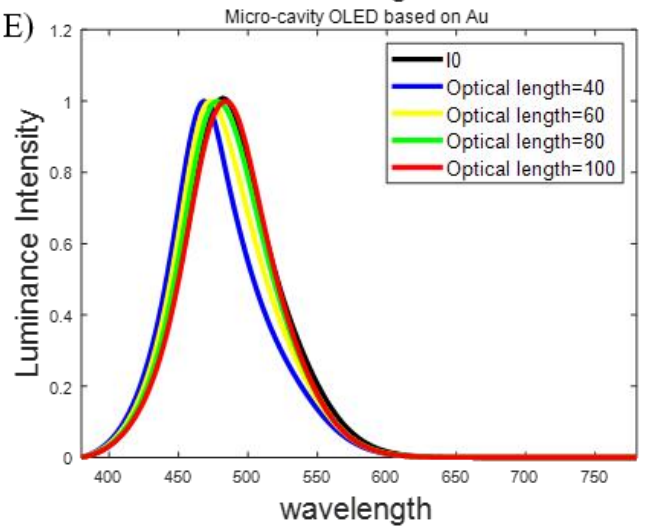

B).
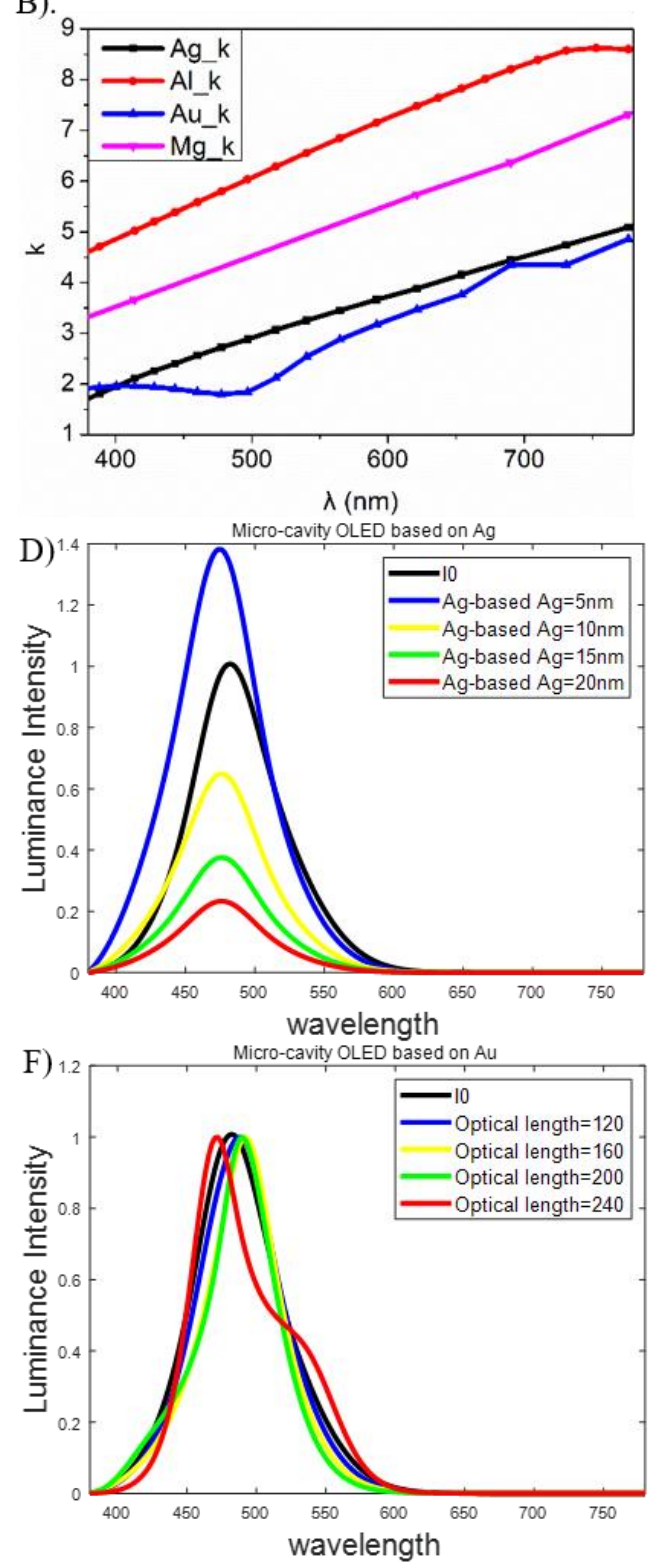

Figure 5. (A) Ordinary refractive indexes of different metals, (B) Extinction coefficients of different metals, (C) Simulated EL spectra of micro-cavity OLEDs based on Au with different thicknesses, (D) Simulated EL spectra of micro-cavity OLEDs based on Ag with different thicknesses, (E,F) Simulated EL spectra of micro-cavity OLEDs based on Au with different optical paths.

Then, the simulated complex dielectric constants $\varepsilon$ and complex refractive index $\tilde{n}$ of $\mathrm{Ag}$ and $\mathrm{Al}$ are shown in Figure $6 \mathrm{~A}, \mathrm{~B}$, in which $\varepsilon_{1}$ and $\varepsilon_{2}$ is the real and the imaginary part of $\varepsilon$, and $n$ and $\kappa$ is the ordinary refractive index and the extinction coefficient, respectively.

According to Equations (6)-(13), Figure 6C-E shows the simulated reflectivity, transmissivity of the Ag layer with $5 \mathrm{~nm}$-, $10 \mathrm{~nm}$-, $15 \mathrm{~nm}$ - and $20 \mathrm{~nm}$-thickness and of $100 \mathrm{~nm}$-thick Al, as well as their phase shifts.

Obviously, thickness affects the optical constants of $\mathrm{Ag}$ and $\mathrm{Al}$ at different wavelengths. With an increasing thickness, the transmissivity of the Ag thin film decreases, while it has a perfect transmissivity at the range of 400-500 nm. Total reflection occurs for a $100 \mathrm{~nm}$-thick Al layer, and its phase shift is unchanged during the visible region, which means that the phase shift stays the same when it reaches a certain thickness. Then, we measured the transmissivity of Ag with different thicknesses combined 
with ITO, as shown in Figure 7E, in order to make sure that the electroluminescence of OLEDs could be passed through. Apparently, there is a difference in transparence between Ag in its solid state by simulation and the Ag thin film in practice. It is found that the whole detected transmissivity decreases slightly with an increasing Ag thickness when combined with ITO in Figure 7E which is different from simulated transmissivity of Ag with different thickness in Figure 6D. It may be due to the light interference between the Ag and ITO layer.
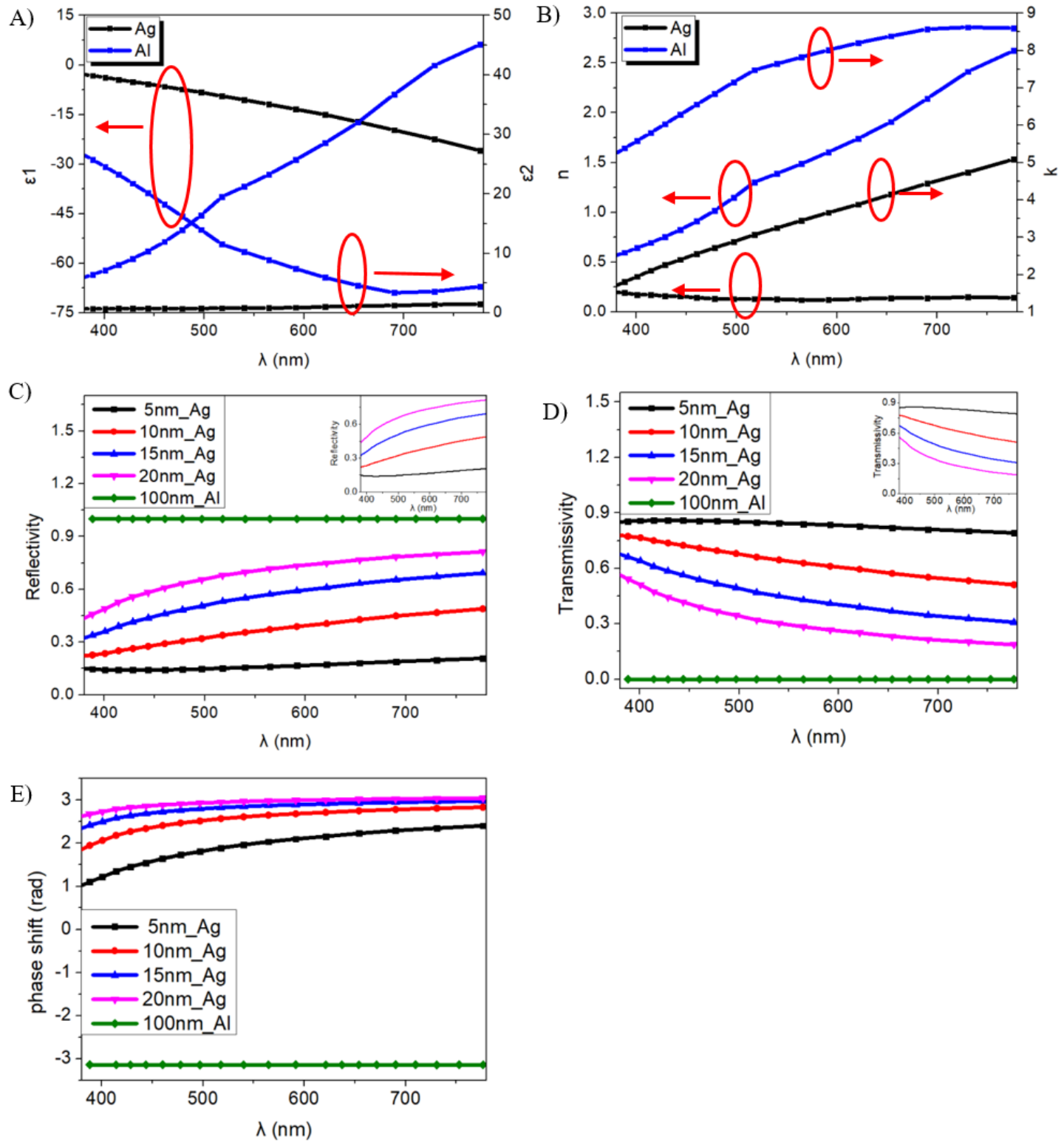

Figure 6. (A) Dielectric function, (B) refractive indexes, (C) simulated reflectivity, (D) simulated transmissivity of $\mathrm{Ag}$ and $\mathrm{Al}$ and $(\mathbf{E})$ the simulated phase shift from $\mathrm{Ag}$ and $\mathrm{Al}$.

Figure 7A describes the PL spectra with a PL peak of $482 \mathrm{~nm}$ of the emission layer of mCBP:SpiroAC-TRZ. Figure 7B shows the simulated EL spectra of the micro-cavity OLEDs with an Ag layer with different optical paths and of ITO-based OLEDs whose EL spectra were assumed to be similar to the PL spectra of mCBP:SpiroAC-TRZ due to the high transmissivity of ITO and the fact that it was normalized. With the highest transmissivity of all Ag layers, $5 \mathrm{~nm}$-thick Ag enhances the luminance intensity because of its reflectivity contrast with ITO. By increasing the thickness of the Ag layer, the transmissivity decreases and the reflectivity rises to a similar degree, as shown in 
Figure 6C,D, while the forward emission of micro-cavity OLEDs with a 20 nm-thick Ag layer decreases to the minimum in simulated OLEDs. In addition, the phase shift also influences the intensity greatly. Figure 7C shows the simulated luminance intensity of $480 \mathrm{~nm}$ in micro-cavity OLEDs with L1 and L2 changing from 30 to 200, representing the optical path from EML to the cathode and anode, respectively. The surface and its top view in Figure 7C,D represent the intensity of micro-cavity OLEDs with a $10 \mathrm{~nm}$-thick Ag layer, and the dark plane in this figure describes the intensity of ITO-based OLEDs. They show that the cavity length and the position of EML influence the spectra and intensity of the forward emission of OLEDs. There are two intersection lines between the dark plane and the surface of the emission of micro-cavity OLEDs shown in Figure 7D. The diagonal line from the coordinate point $(50,50)$ to $(200,200)$ also has two intersection points with two intersection lines. When comparing the $x, y$ coordinate values of the intersection points, we can find that the optical path L1 has a stronger impact on the intensity than the optical path L2 does.
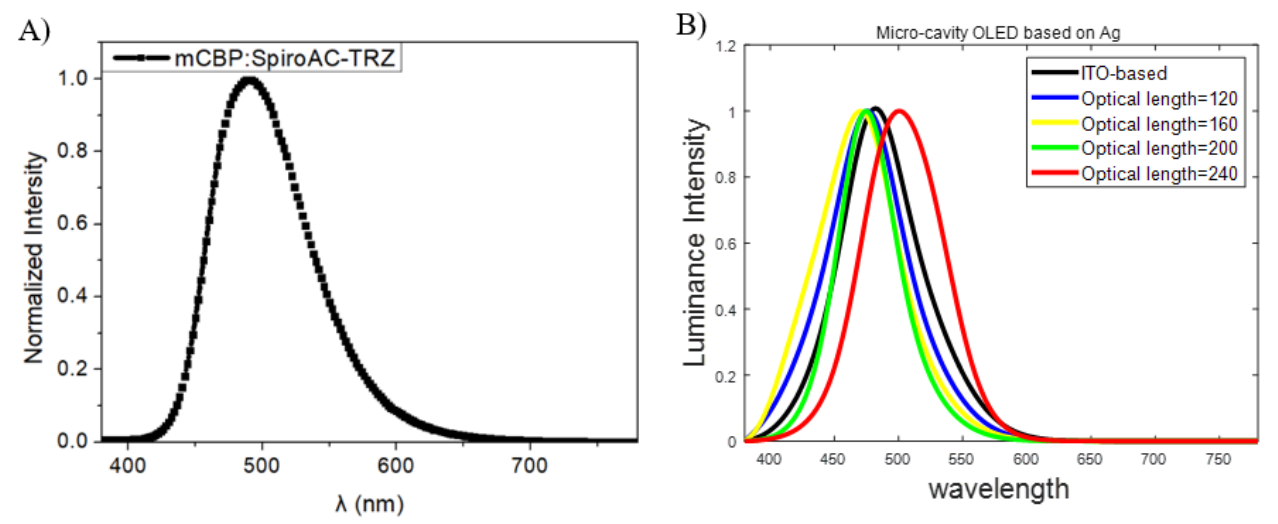

C)

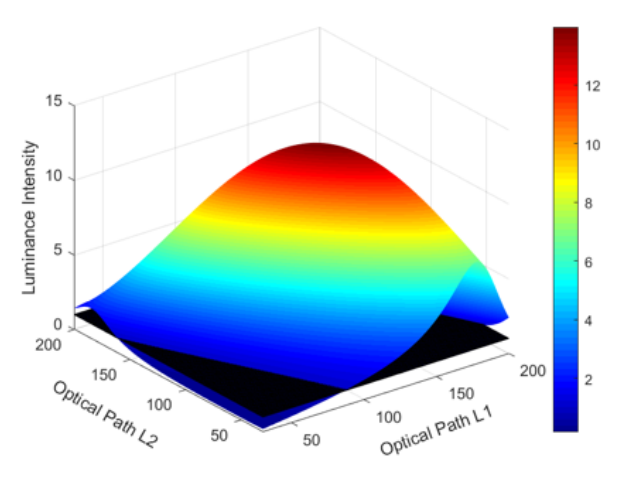

E)

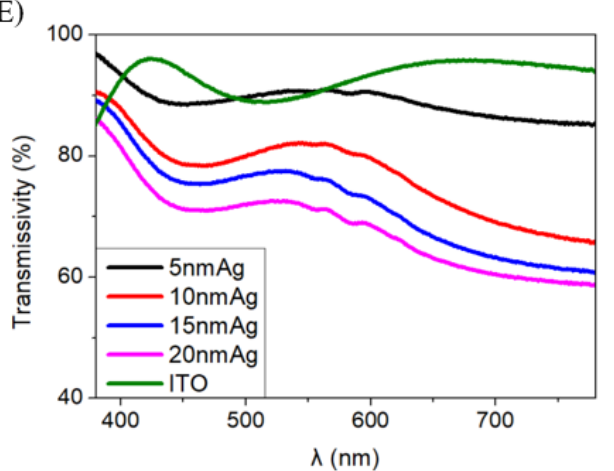

D)

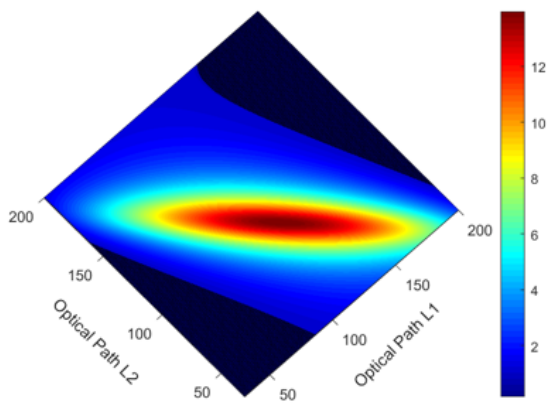

Figure 7. (A) PL spectra of mCBP doped with SpiroAC-TRZ, (B) Simulated EL spectra of micro-cavity OLEDs at different optical lengths, (C) Simulated luminance intensity of $480 \mathrm{~nm}$ in micro-cavity OLEDs with different optical paths L1 and L2, contrasted with ITO-based OLEDs (D) and its top view, and (E) the measured transmissivity of $\mathrm{Ag}(\mathrm{x} \mathrm{nm}, \mathrm{x}=5,10,15,20) / \mathrm{ITO}$ and ITO. 


\subsection{Deep Blue OLEDs Using Micro-Cavity}

The transmission of the forward Ag layer recombined with ITO was detected, as shown in Figure 7F, to make sure that the electroluminescence of OLEDs could be passed through. The Ag thickness is $5 \mathrm{~nm}, 10 \mathrm{~nm}, 15 \mathrm{~nm}$ and $20 \mathrm{~nm}$, respectively. In terms of forward emission, combined with Figure 5D, especially in the blue region, a better transmission and lower extinction coefficient are required. Meanwhile, the higher reflectivity and phase shift contribute to a constructive interference, which results in an extra enhanced intensity. However, the low wetting capability of the $5 \mathrm{~nm}$-thick $\mathrm{Ag}$ layer causes un-continuous $\mathrm{Ag}$ islands. In view of the transparence and electric property, a $10 \mathrm{~nm}$-thick $\mathrm{Ag}$ thin film is the appropriate choice. The devices used here are in a configuration of ITO/Ag $(10 \mathrm{~nm}) / \mathrm{HAT}-\mathrm{CN}(20 \mathrm{~nm}) / \mathrm{TCTA}(20 \mathrm{~nm}) / \mathrm{mCBP}: S p i r o A C-T R Z(w t .10 \%, \mathrm{xnm}) / \mathrm{TPBi}(20 \mathrm{~nm}) / \mathrm{LiF}$ $(1 \mathrm{~nm}) / \mathrm{Al}(100 \mathrm{~nm})$. The control devices are: ITO/ HAT-CN $(20 \mathrm{~nm}) / \mathrm{TCTA}(20 \mathrm{~nm}) / \mathrm{mCBP}: S p i r o A C-T R Z$ (wt.10\%, xnm)/TPBi $(20 \mathrm{~nm}) / \mathrm{LiF}(1 \mathrm{~nm}) / \mathrm{Al}(100 \mathrm{~nm})$.

As shown in Figure 8, the spectrum of devices is based on ITO with different thicknesses of EML from $25 \mathrm{~nm}$ to $50 \mathrm{~nm} ; 75 \mathrm{~nm}$ changes little, and is almost same with an approximate EL peak and FWHM at $487 \mathrm{~nm}$ and $87 \mathrm{~nm}$, respectively. Apparently, the EL peak of devices with the micro-cavity based on ITO/Ag (10 nm) blue-shifts to $479 \mathrm{~nm}$, then turns to the red shift to $487 \mathrm{~nm}$ and $493 \mathrm{~nm}$ along with an increasing EML thickness. The shifting trend of EL peaks is consistent with the simulation results. It turns out that Ag thin film as anode leads to the blue-shifting of OLEDs' spectrum, due to its reflectivity and obvious different refractive index when compared with organic layers, which brings about the phase shifting according to the simulation result shown in Figure 6E. The increased thickness of EML makes the spectrum red-shift because the optical paths L1 and L2 get longer. The reflected light contributes to the interference light, which is influenced and regulated by the joint effect of the phase shift and optical path, forming the EL spectrum. In addition to the peak blue shift, the FWHM of the spectrum of the micro-cavity OLED with an EML with a 25-nm thickness turns to $69.8 \mathrm{~nm}$ and is narrower than that of ITO-based OLEDs. When the spectrum red-shifts, its FWHM changes from $69.8 \mathrm{~nm}$ to $75.5 \mathrm{~nm}$ and $83.2 \mathrm{~nm}$, respectively. All of the above show that the micro-cavity helps to suppress certain wavelengths and obtain a saturated deep blue by regulating the spectra.

A)

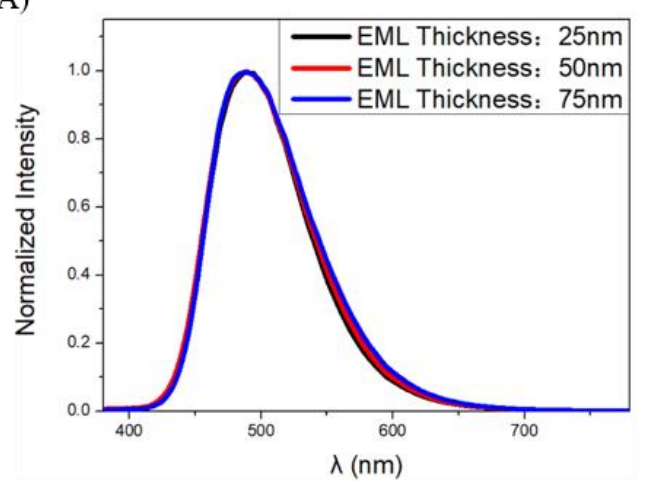

B).

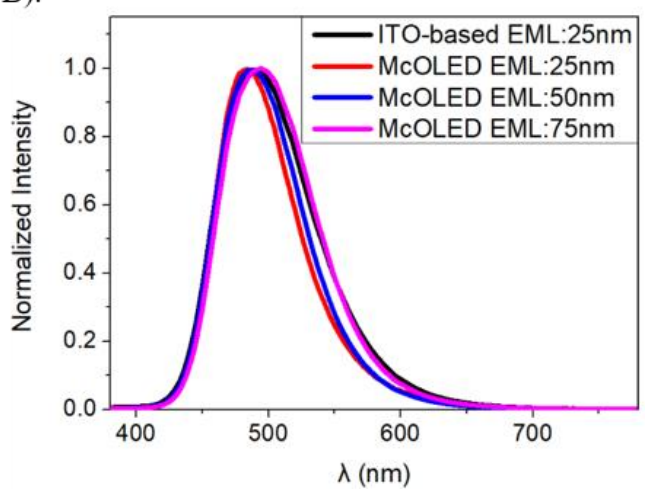

Figure 8. (A) EL Spectra of ITO-based OLEDs with EML with 25, 50 and $75 \mathrm{~nm}$ thicknesses, and (B) EL Spectra of micro-cavity OLEDs (McOLED) with EML with 25, 50 and $75 \mathrm{~nm}$ thicknesses.

As shown in Figure 9, compared to the control devices based on ITO with a 50-nm EML, the $V_{\text {on }}$ of the micro-cavity device increases and the luminance decreases, which is due to the extra resistance and low transmission resulting from the existence of the Ag thin film. We speculate that the contact between ITO and Ag results in the poor carrier injection, while the optical loss is caused by the surface plasma effect and defects or traps that Ag brings in for its ultrathin thickness. The $10 \mathrm{~nm}$-thick Ag thin film can cause a point discharge because of its non-uniform morphology. Therefore, although the Ag thin film is easy to fabricate, the thinner micro-cavity OLEDs' structure is easily broken down under 
a high current. Thus, the spectrum blue-shifting with the increased thickness of the Ag layer needs further investigation in such micro-cavity OLEDs in order to optimize their electroluminescence.

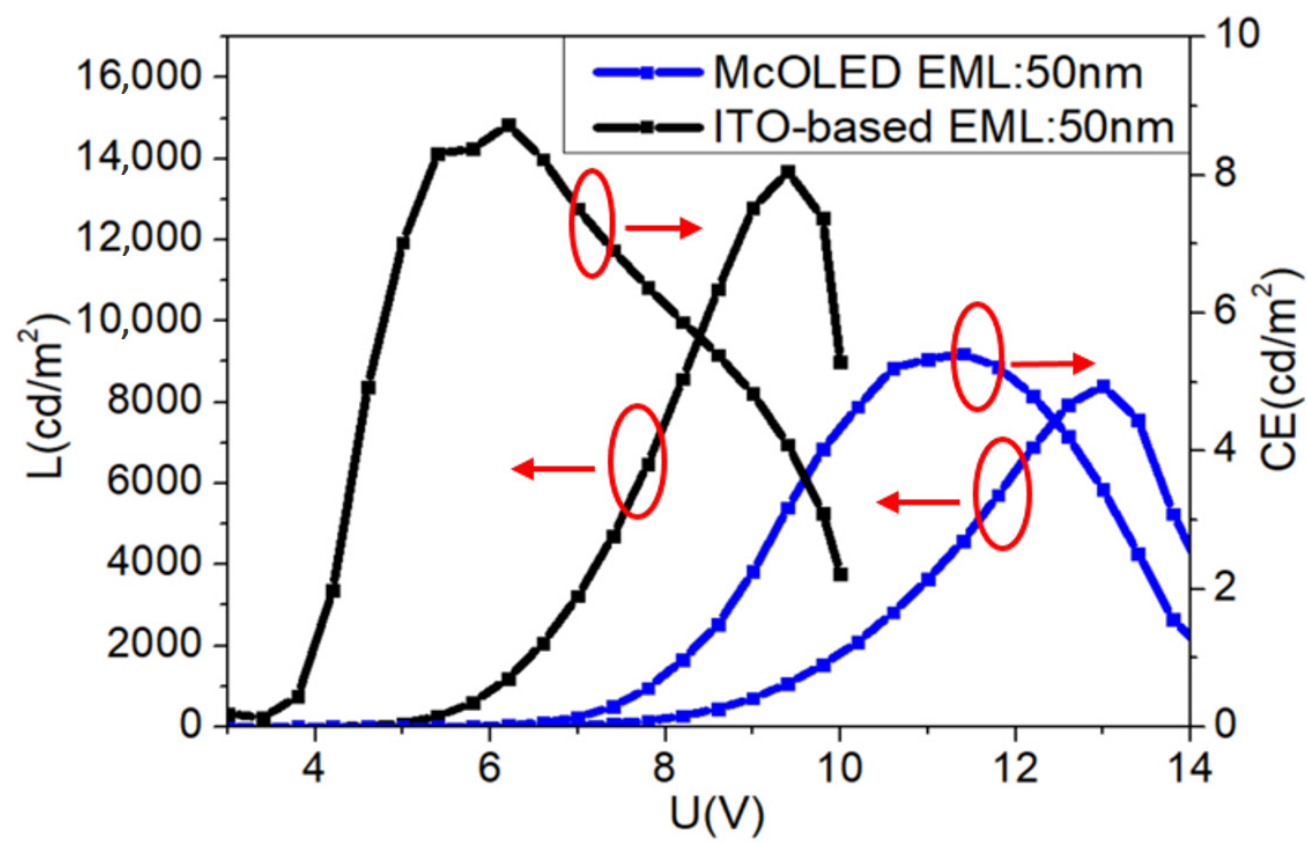

Figure 9. Current density-voltage-luminance of the ITO-based OLED and micro-cavity OLEDs.

The electroluminescence device performances of ITO-based OLEDs and micro-cavity OLEDs are shown in Table 1. It shows that the micro-cavity structure obviously regulates the spectra of OLEDs, as the simulation shows. As for the intensity, EQE and CE, in order to establish a microcavity in the micro-cavity model in these OLEDs, the Ag electrode has the potential to improve the performance of OLEDs because of its optical properties and induced microcavity effect. However, there turns out to be a suppression of the intensity and current efficiency, and according to our experiments and analysis, this is mainly owing to defects and traps resulting from the rough morphology of the Ag film and SPP (surface plasmon polaritons) of Ag. In addition, the inferior adhesion of Ag to ITO is another problem related to the issue of a better performance. Besides these, owing to the low transparency of the $10 \mathrm{~nm}$-thick Ag electrode, the luminance of the forward emission decreases with decreasing efficiencies. Thus, it is feasible to improve the performances of devices dramatically and change the spectra in the blue region with a $5 \mathrm{~nm}$-thick Ag layer if we settle the problem of the point discharge.

Table 1. The electroluminescence device performances of ITO-based OLEDs and micro-cavity OLEDs.

\begin{tabular}{cccccc}
\hline Device & EML (nm) & Lmax (cd) & CE (cd/A) & Peak Wavelength $(\mathbf{n m})$ & FMWH $(\mathbf{n m})$ \\
\hline ITO-based 1 & 25 & 5100 & 3.64 & 487 & 86 \\
ITO-based 2 & 50 & 13,684 & 8.73 & 487 & 89 \\
ITO-based 3 & 75 & 16,835 & 12 & 481 & 87 \\
McOLED 1 & 25 & 1245.4 & 0.683 & 479 & 69.8 \\
McOLED 2 & 50 & 8413.8 & 5.41 & 487 & 75.5 \\
McOLED 3 & 75 & 8450 & 5.62 & 493 & 83.2 \\
\hline
\end{tabular}

\section{Materials and Methods}

\subsection{Materials}

10-(4-(4,6-diphenyl-1,3,5-triazin-2-yl)phenyl)-10H-spiro [acridine-9,9'-fluorene] (SpiroAC-TRZ) and 3,3'-Di(9H-carbazol-9-yl) biphenyl(m-CBP) were purchased from Taiwan Luminescence Technology Corp. 
Dipyrazino[2,3-f:2', 3'-h ]quinoxaline-2,3,6,7,10,11-hexacarbonitrile (HAT-CN), di-[4-(N,N -di-p -tolyl-amino)-phenyl]cyclohexane (TAPC) and 1,3,5-tris(1-phenyl-1H-benzimidazol-2-yl)benzene (TPBi) were purchased from Xi'an Polymer Light Technology Corp.

\subsection{Device Fabrication}

All organic and inorganic materials used in this paper were commercially obtained without further purification. The devices used in our work were fabricated on patterned ITO-coated glass substrates with a sheet resistance of $15 \Omega / \mathrm{sq}$, which were routinely cleaned in an ultrasonic bath with distilled water and ethyl alcohol. Then, the substrates were treated by $\mathrm{O}_{2}$ plasma under conditions of $1.3 \times 10^{-2} \mathrm{~Pa}$ at $75 \mathrm{~W}$ for $3 \mathrm{~min}$. All the layers were grown by thermal evaporation method at the base pressure of $<5 \times 10^{-4} \mathrm{~Pa}$. The deposition rate was $0.1 \mathrm{~nm} / \mathrm{s}$ for all organic layers and $0.01 \mathrm{~nm} / \mathrm{s}$ for the $\mathrm{LiF}$ layer. Then, the $\mathrm{Al}$ cathode was deposited at a rate of $0.2 \mathrm{~nm} / \mathrm{s}$ directly without opening the vacuum chamber. All the deposition rates and film thicknesses were monitored by a quartz crystal oscillator.

\subsection{Device Measurements}

The current density-voltage-luminance $(\mathrm{J}-\mathrm{V}-\mathrm{L})$ characteristics and electroluminescence spectra of the OLED devices were measured simultaneously using a programmable source meter (Keithley model 2400) and a luminance meter/spectrometer (Photo Research PR655). All the measurements were conducted in ambient air. The transparencies of the ITO and Ag thin films were measured using an ultraviolet-visible spectrophotometer (UV-3100 spectrophotometer).

\section{Conclusions}

In summary, we make a theoretical analysis of micro-cavity OLEDs, and run a simulation of it. Our findings show that it is feasible to adopt a micro-cavity structure for regulating the visible spectra of OLEDs. We can get an expected blue shift by optimizing the optical path of devices and the phase shift from metallic electrodes. The simulation shows that transmission is essential for the forward emission of blue OLEDs, while reflectivity with the phase shift makes it possible to obtain a good performance. By performing experiments, we validated that using Fabry-Pérot interference in the micro-cavity can enhance the wavelengths we expected and suppress other wavelengths in order to regulate intrinsic EL peak blue shift precisely. By tuning the micro-cavity modes, the EL peak wavelength of blue OLEDs has a redshift from $479 \mathrm{~nm}$ to $493 \mathrm{~nm}$, with an FWHM from $69.8 \mathrm{~nm}$ to $83.2 \mathrm{~nm}$, compared to devices without a micro-cavity whose approximate EL peak wavelength and FWHM are $487 \mathrm{~nm}$ and $87 \mathrm{~nm}$, respectively. However, in this paper, Ag-based micro-cavity OLEDs perform less well than DBR-based ones, for example in relation to a limited blue shift in the spectra and a suppression in intensity and current efficiency. As for mass production, Ag has the potential to be the alternative for ITO due to its good mechanical/electrical/optical properties and the fact that it is easy to fabricate. Thus, we plan to further investigate how to improve the performance of micro-cavity OLEDs based on Ag electrodes.

Author Contributions: Data curation, J.J., Investigation, W.Z., J.C. and J.J., Funding acquisition, S.Z., Project administration, S.Z., Methodology, B.Q. and J.J., Supervision, D.S., Resources, Z.X., Writing-original draft, J.J., Writing-review \& editing, S.Z. All authors have read and agreed to the published version of the manuscript.

Funding: This study was supported by the National Key Research and Development Program of China under Grant No. 2016YFB0401302 and the National Natural Science Foundation of China under Grant No. 61704007 and No. 61775013.

Conflicts of Interest: The authors declare no conflict interests. 


\section{References}

1. Tang, C.W.; Vanslyke, S.A. Organic electroluminescent diodes. Appl. Phys. Lett. 1987, 51, 913-915. [CrossRef]

2. Tang, C.W.; Vanslyke, S.A.; Chen, C.H. Electroluminescence of doped organic thin films. J. Appl. Phys. 1989, 65, 3610-3616. [CrossRef]

3. Baldo, M.A.; Thompson, M.E.; Forrest, S.R. High-efficiency fluorescent organic light-emitting devices using a phosphorescent sensitizer. Nature 2000, 403, 750-753. [CrossRef] [PubMed]

4. D'Andrade, B.W.; Holmes, R.; Forrest, S.R. Efficient Organic Electrophosphorescent White-Light-Emitting Device with a Triple Doped Emissive Layer. Adv. Mater. 2004, 16, 624-628. [CrossRef]

5. Gurin, N.T.; Paksyutov, K.V.; Terent'ev, M.A.; Shirokov, A.V. CaO center dot Al2O3 center dot SiO2: Eu Phosphors for Violet/Ultraviolet-to-White Radiation Conversion. Tech. Phys. 2012, 57, 308-310. [CrossRef]

6. Gao, Z.; Cheng, G.; Shen, F.; Zhang, S.; Zhang, Y.; Lu, P.; Ma, Y. Highly efficient deep blue light emitting devices based on triphenylsilane modified phenanthro[9, 10- d ]imidazole. Laser Photon- Rev. 2013, 8. [CrossRef]

7. Shin, H.; Ha, Y.H.; Kim, H.; Kim, R.; Kwon, S.-K.; Kim, Y.-H.; Kim, J.-J. Controlling Horizontal Dipole Orientation and Emission Spectrum of Ir Complexes by Chemical Design of Ancillary Ligands for Efficient Deep-Blue Organic Light-Emitting Diodes. Adv. Mater. 2019, 31, e1808102. [CrossRef]

8. Feng, W.; Su, Q.; Ma, Y.; Dzolic, Z.; Huang, F.; Wang, Z.; Chen, S.; Tang, B.Z. Tetraphenylbenzosilole: An AIE Building Block for Deep-Blue Emitters with High Performance in Nondoped Spin-Coating OLEDs. J. Org. Chem. 2019, 85, 158-167. [CrossRef]

9. Rajamalli, P.; Chen, D.; Li, W.; Samuel, I.D.W.; Cordes, D.B.; Slawin, A.M.Z.; Zysman-Colman, E. Enhanced thermally activated delayed fluorescence through bridge modification in sulfone-based emitters employed in deep blue organic light-emitting diodes. J. Mater. Chem. C 2019, 7, 6664-6671. [CrossRef]

10. Qiu, X.; Ying, S.; Wang, C.; Hanif, M.; Xu, Y.; Li, Y.; Zhao, R.; Hu, D.; Ma, D.; Ma, Y. Novel 9,9-dimethylfluorene-bridged D- $\pi$-A-type fluorophores with a hybridized local and charge-transfer excited state for deep-blue electroluminescence with CIEy 0.05. J. Mater. Chem. C 2019, 7, 592-600. [CrossRef]

11. Jia, Y.; Zhang, Y.; Fan, S.; Wu, S.; Zhao, X.; Wang, S.; Li, X. A novel bipolar carbazole/ phenanthroimidazole derivative for high efficiency nondoped deep-blue organic light-emitting diodes. Org. Electron. 2019, 64, 259-265. [CrossRef]

12. Kondo, Y.; Yoshiura, K.; Kitera, S.; Nishi, H.; Oda, S.; Gotoh, H.; Sasada, Y.; Yanai, M.; Hatakeyama, T. Narrowband deep-blue organic light-emitting diode featuring an organoboron-based emitter. Nat. Photonic 2019, 13, 678-682. [CrossRef]

13. Jou, J.-H.; Li, J.-L.; Sahoo, S.; Dubey, D.K.; Yadav, R.A.K.; Joseph, V.; Thomas, K.R.J.; Wang, C.-W.; Jayakumar, J.; Cheng, C.-H. Enabling a 6.5\% External Quantum Efficiency Deep-Blue Organic Light-Emitting Diode with a Solution-Processable Carbazole-Based Emitter. J. Phys. Chem. C 2018, 122, 24295-24303. [CrossRef]

14. Byeon, S.Y.; Lee, D.R.; Yook, K.S.; Lee, J.Y. Recent Progress of Singlet-Exciton-Harvesting Fluorescent Organic Light-Emitting Diodes by Energy Transfer Processes. Adv. Mater. 2019, 31. [CrossRef] [PubMed]

15. Chen, W.-C.; Yuan, Y.; Wu, G.-F.; Wei, H.-X.; Tang, L.; Tong, Q.-X.; Wong, F.-L.; Lee, C. Staggered Face-to-Face Molecular Stacking as a Strategy for Designing Deep-Blue Electroluminescent Materials with High Carrier Mobility. Adv. Opt. Mater. 2014, 2, 626-631. [CrossRef]

16. Lee, J.-H.; Chen, C.-H.; Lee, P.-H.; Lin, H.-Y.; Leung, M.-K.; Chiu, T.-L.; Lin, C.-F. Blue organic light-emitting diodes: Current status, challenges, and future outlook. J. Mater. Chem. C 2019, 7, 5874-5888. [CrossRef]

17. Xu, Z.; Tang, B.Z.; Wang, Y.; Ma, D. Recent advances in high performance blue organic light-emitting diodes based on fluorescence emitters. J. Mater. Chem. C 2020, 8, 2614-2642. [CrossRef]

18. Liu, Y.; Li, C.; Ren, Z.; Yan, S.; Bryce, M.R. All-organic thermally activated delayed fluorescence materials for organic light-emitting diodes. Nat. Rev. Mater. 2018, 3, 18020. [CrossRef]

19. Ji, W.; Zhang, L.; Tianyu, Z.; Xie, W.; Zhang, H. High-contrast and high-efficiency microcavity top-emitting white organic light-emitting devices. Org. Electron. 2010, 11, 202-206. [CrossRef]

20. Schwab, T.; Schubert, S.; Müller-Meskamp, L.; Leo, K.; Gather, M.C. Eliminating Micro-Cavity Effects in White Top-Emitting OLEDs by Ultra-Thin Metallic Top Electrodes. Adv. Opt. Mater. 2013, 1, 921-925. [CrossRef] 
21. Li, J.-Y.; Chen, S.-P.; Siao, H.-J.; Wu, J.-H.; Chen, G.-Y.; Chen, C.-C.; Ho, S.-Y.; Lin, Y.-P.; Hsu, H.-H.; Lin, J.-S.; et al. Significant color space blue-shift of green OLED emitter with sustaining lifetime and substantial efficiency enhancement. Appl. Phys. Lett. 2017, 111, 093301. [CrossRef]

22. Genco, A.; Giordano, G.; Carallo, S.; Accorsi, G.; Duan, Y.; Gambino, S.; Mazzeo, M. High quality factor microcavity OLED employing metal-free electrically active Bragg mirrors. Org. Electron. 2018, 62, 174-180. [CrossRef]

23. Han, J.H.; Kim, D.; Lee, T.-W.; Jeong, E.G.; Lee, H.S.; Choi, K.C. Ultra-High-Resolution Organic Light-Emitting Diodes with Color Conversion Electrode. ACS Photonic 2018, 5, 1891-1897. [CrossRef]

24. Christogiannis, N.; Somaschi, N.; Michetti, P.; Coles, D.M.; Savvidis, P.G.; Lagoudakis, P.G.; Lidzey, D.G. Characterizing the Electroluminescence Emission from a Strongly Coupled Organic Semiconductor Microcavity LED. Adv. Opt. Mater. 2013, 1, 503-509. [CrossRef]

25. Zhang, J.; Song, J.; Zhang, H.; Ding, H.; Guo, K.; Wei, B.; Zheng, Y.-Q.; Zhang, Z. Sunlight-like white organic light-emitting diodes with inorganic/organic nanolaminate distributed Bragg reflector (DBR) anode microcavity by using atomic layer deposition. Org. Electron. 2016, 33, 88-94. [CrossRef]

26. Meister, S.; Bruckner, R.; Sūdžius, M.; Fröb, H.; Leo, K. Optically pumped lasing of an electrically active hybrid OLED-microcavity. Appl. Phys. Lett. 2018, 112, 113301. [CrossRef]

27. Lin, C.-L.; Lin, H.-W.; Wu, C.-C. Examining microcavity organic light-emitting devices having two metal mirrors. Appl. Phys. Lett. 2005, 87, 21101. [CrossRef]

28. Guo, T.-F.; Yang, F.-S.; Tsai, Z.-J.; Feng, G.-W.; Wen, T.-C.; Hsieh, S.-N.; Chung, C.-T.; Wu, C.-I. High-brightness top-emissive polymer light-emitting diodes utilizing organic oxide/Al/Ag composite cathode. Appl. Phys. Lett. 2006, 89, 051103. [CrossRef]

29. Peng, H.; Sun, J.; Zhu, X.; Yu, X.; Wong, M.; Kwok, H.-S. High-efficiency microcavity top-emitting organic light-emitting diodes using silver anode. Appl. Phys. Lett. 2006, 88, 73517. [CrossRef]

30. Poitras, D.; Kuo, C.-C.; Py, C. Design of high-contrast OLEDs with microcavity effect. Opt. Express 2008, 16, 8003-8015. [CrossRef]

Sample Availability: Samples of the compounds are not available from the authors.

(C) 2020 by the authors. Licensee MDPI, Basel, Switzerland. This article is an open access article distributed under the terms and conditions of the Creative Commons Attribution (CC BY) license (http://creativecommons.org/licenses/by/4.0/). 